\title{
ESTILOS COGNITIVOS DE ESTUDANTES DE PSICOLOGIA: EXPERIÊNCIA EM INICIAÇÃO CIENTÍFICA E SÉRIES FREQÜENTADAS*
}

\section{COGNITIVE STYLES OF PSYCHOLOGY COLLEGE STUDENTS: SCIENTIFIC INICIATION AND YEARS ATTENDED}

\author{
Isabel Cristina Dib BARIAN|** \\ (Pontifícia Universidade Católica de Campinas) \\ Acácia A. Angeli dos SANTOS \\ (Universidade São Francisco)
}

\begin{abstract}
RESUMO
Neste estudo, estilos cognitivos são compreendidos como características relativamente estáveis da estrutura cognitiva de uma pessoa. Teve-se como objetivos: descrever e comparar os estilos cognitivos de estudantes de Psicologia, com e sem experiência em iniciação científica (IC) e verificar se há variação na predominância dos estilos em função das séries freqüentadas. Os dados foram coletados com 447 universitários de um Curso de Psicologia, por meio de uma escala que descreve estilos cognitivos. Comparando os estilos dos estudantes com e sem experiência em IC, observou-se que os bolsistas de IC apresentam menos Convergência de Pensamento do que os demais alunos, são mais Divergentes e menos Dependentes de Campo. A análise entre as séries sugere que do início ao final do curso ocorre diminuição dos estilos Convergência e Dependência de Campo. Este estudo permite concluir que os estilos cognitivos não são estáveis, mas passíveis de modificação ao longo dos anos de universidade.
\end{abstract}

Palavras-chave: estilo cognitivo; estudantes universitários; iniciação científica.

\begin{abstract}
Cognitive styles are regarded as fairly stable characteristics of a person's cognitive structure. This research aimed at describing and comparing
\end{abstract}

\footnotetext{
(*) Versão condensada de parte da Tese de Doutorado de Isabel Cristina Dib Bariani, defendida em 06-02-98, FE -UNICAMP, sob a orientação da Prota. Dra. Acácia A. Angeli dos Santos.

${ }^{(*)}$ Endereço para correspondência: PUC-Campinas, Instituto de Psicologia e Fonoaudiologia, Rua Marechal Deodoro, 1117, Centro, CEP 13020-904, Campinas, SP. E-mail: dbariani@uol.com.br
} 


\begin{abstract}
the cognitive styles of Psychology college students who have been or not scientific initiation scholars and verifying whether the predominant styles vary according to the year the students are taking. The data was collected through a scale developed aiming at the description of cognitive styles and the sample consisted of 447 college students from Psychology school. Among the students with and without experience in scientific initiation programs it is observed that the scientific initiation scholars are more Divergent and less Field Dependents. The analysis of the different classes suggests that from its beginning to the end it occurs a decrease of Convergent and Dependence Field styles. This study provides support to the conclusion that cognitive styles are not stable but liable to change over the college years.
\end{abstract}

Key words: cognitive style, college students; scientific initiation.

Uma diversidade de variáveis psicológicas são apontadas como mediadoras do processo ensino-aprendizagem e, dentre elas, os estilos cognitivos têm sido objeto de estudos e discussões por pesquisadores de diversos países.

Quanto à definição, uma grande variedade de concepções de estilos cognitivos é encontra da na literatura. Messick (1984) destaca que, embora a diversidade de caracterização de estilos cognitivos seja fonte de confusão nesse campo de estudos, as várias concepções existentes são sobrepostas ao invés de mutuamente exclusivas.

Apesar da multiplicidade de definições, os estudiosos têm concordado em um aspecto: os diferentes conceitos não implicam níveis de habilidade, capacidade, ou inteligência. Não se trata de uma habilidade em si mesma e sim do modo preferencial de alguém usar habilidades, não havendo estilos bons ou maus, mas apenas diferentes (Messick, 1984; Palmer, 1991; Thompson e Crutchlow, 1993).

Os estilos cognitivos podem ser compreendidos como formas relativamente estáveis referentes às características da estrutura cognitiva de uma pessoa, que são definidas, em parte, por fatores biológicos, sendo influenciadas pela cultura, ou seja, são modificadas a partir da influência direta ou indireta de novos eventos. Eles denotam tendências diferenciadas básicas nas formas de apreender e relacionar os dados da realidade e de elaborar conclusões sobre eles. Dizem respeito à forma e não ao conteúdo do que se pensa, sabe, percebe, lembra, aprende e decide (Bariani, 1998).

Dentre as dimensões de estilos cognitivos identificadas e descritas, as mais discutidas e Investigadas são: Dependência-Independência de Campo, Reflexividade-Impulsividade de Resposta, Divergência-Convergência de Pensamento e Holista-Serialista (a primeira tem sido mais freqüentemente abordada). Estas quatro dimensões são assim descritas:

\section{Dependência - Independência de Campo}

Dependência de campo: Indivíduos com campo dependente contam com uma estrutura externa de referência e assim preferem conteúdo e seqüência previamente organizados; requerem mais reforçamento extrínseco. São hábeis em situações que demandam percepção pessoal e habilidades interpessoais; preferem uma interação professor-aluno mais informal; relutam em dar "feedback" crítico.

Independência de campo: As pessoas com campo independente contam com uma estrutura interna de referência, preferindo envolver-se na organização e seqüenciação de conteúdos; respondem a reforçamento intrínseco. São mais autônomas e com grande 
confiança em si mesmas. Saem-se melhor em situações que requerem uma análise impessoal; facilmente corrigem o outro e expõem porque errou; preocupam-se mais com o conteúdo do que com a interação professor-aluno.

\section{Impulsividade - Reflexividade de Resposta}

Impulsividade: Pessoas impulsivas detêmse pouco em ponderação e organização prévia a uma resposta.

Reflexividade: São consideradas as pessoas cujos pensamentos são mais organizados, seqüenciados e que fazem ponderação prévia a uma resposta.

\section{Convergência - Divergência de Pensamento}

Convergência de Pensamento: É identificada com o raciocínio e o pensamento lógico. As pessoas de pensamento convergente são hábeis em lidar com problemas que requerem uma clara resposta convencional (uma solução correta), a partir das informações fornecidas. Preferem problemas formais e tarefas mais bem estruturadas, que demandam mais as habilidades lógicas. São inibidos emocionalmente, sendo identificados como mais conformistas, disciplinados e conservadores.

Divergência de Pensamento: É associado à criatividade, a respostas imaginativas, originais e fluentes. São os indivíduos que preferem problemas menos estruturados, que são hábeis em tratar de problemas que demandam a generalização de várias respostas igualmente aceitáveis, onde a ênfase é na quantidade, variedade e originalidade das respostas. Socialmente, são considerados como irritadiços, disruptivos e até ameaçadores.

\section{Holista - Serialista}

Holista: Indivíduos que dão maior ênfase ao contexto global desde o início de uma tarefa; preferem examinar uma grande quantidade de dados, buscando padrões e relações entre eles. Usam hipóteses mais complexas, às quais combinam diversos dados.

Serialista: Pessoas que dão maior ênfase a tópicos separados e em seqüências lógicas, buscando posteriormente padrões e relações no processo, para confirmar ou não suas hipóteses. Assim, utilizam-se de uma abordagem lógico-linear (partindo de uma hipótese mais simples para a próxima, com complexidade crescente).

Há indícios na literatura de que diferentes estilos cognitivos são imbricados, embora haja pouca evidência de correlações empíricas entre os estilos. Riding e Cheema (1991) consideram que os indivíduos Dependentes de Campo são mais atentos à idéia ou imagem geral, ao conjunto, e portanto são mais Holistas. Por outro lado,os Independentes de Campo voltam a sua atenção para pequenos elementos informativos. Estes últimos são, ainda, considerados como menos criativos, sendo portanto mais Convergentes, enquanto os sujeitos Campo Dependente têm um pensamento mais Divergente. Os Divergentes são também considerados Impulsivos. Por outro lado, Bar-Haïm (1988) sugere estas mesmas imbricações, com exceção dos estilos Dependência-Independência de Campo, os quais são associados aos outros estilos de maneira inversa à proposta pelos autores citados acima.

A revisão da literatura permitiu a identificação de muitas pesquisas interessadas especialmente nas implicações educacionais dos estilos cognitivos (como, por exemplo, Halpin e Peterson, 1986; Abouserie, Moss e Barasi, 1992; Riding e Sadler-Smith, 1992; Allinson, Hayes e Davis, 1994; Wooten e Barner, 1994; Riding e Wright, 1995).

Os resultados desses estudos sugerem que, embora não sejam facilmente modificáveis, os estilos cognitivos são passíveis de alteração em função de programas de treinamento e práticas educativas. Sua influência é prevista 
em, pelo menos, quatro aspectos do processo ensino- aprendizagem: o planejamento de currículos, os métodos instrucionais, os métodos de avaliação e a orientação a estudantes.

Os estilos cognitivos também parecem ser importantes ao se pensar, particularmente no processo de iniciação científica, pois podese traçar um paralelo entre as descrições das diferentes dimensões de estilos cognitivos e as peculiaridades das características pessoais requeridas no pesquisar, apontadas por diversos autores ( tais como: Bachrach, 1972; Moraes, 1986; Gil, 1987; Marques e Cols, 1989; Wood, 1990; Simão, 1994).

Há estudiosos interessados especialmente em investigar as preferências cognitivas e sua relação com o desenvolvimento da atividade de pesquisa. Dentre eles encontram-se Bar-Haïm (1984 e 1988) e Palmer (1991).

No Brasil, a prática da pesquisa científica não tem sido objeto de um grande número de estudos, especialmente quando realizada por universitários. Entretanto, os benefícios diretos e indiretos da iniciação científica para o aluno, seus estudos, suas futuras atividades profissionais, ou como cidadãos, têm sido salientados por diversos autores (como Bazin, 1983; Zakon, 1989; Demo, 1991; Azzi, 1993; Simão, 1994; Almeida, 1995; Bettoi, 1995; Costa, 1995; e Bariani e Dimárzio, 1997).

Com base no exposto, este trabalho dedicou-se aos seguintes objetivos:

- Descrever e comparar os estilos cognitivos preferenciais de estudantes de Psicologia, com e sem experiência em programas de iniciação científica (IC).

- Verificar se há variação na predominância dos estilos em função das séries freqüentadas.

\section{MÉTODO}

Ao se planejar a proposta metodológica deste estudo, ponderou-se sobre a adequação de se adotar uma abordagem longitudinal ou transversal. Indubitavelmente, um estudo longitudinal permitiria que as inferências realizadas a partir dos resultados obtidos tivessem muito mais sustentação. Entretanto, considerando as dificuldades práticas e técnicas de um delineamento longitudinal (como as apontadas por Kerlinger, 1980), que implicaria o acompanhamento de estudantes ao longo de cinco anos, optou-se por um estudo transversal, mesmo considerando que se contaria com uma maior fragilidade nas evidências.

Assim, serviram como informantes desta pesquisa 447 estudantes do Curso de Psicologia da Pontifícia Universidade Católica de Campinas, São Paulo. Esta amostra representava $71 \%$ do total de alunos do curso, sendo que 101 freqüentavam a $1^{\mathrm{a}}$ série, 98 a $2^{\mathrm{a}}$, 90 a $3^{a}, 83$ a $4^{a}$ e 75 a $5^{a}$ série. Dentre eles, 23 tinham experiência em programas de IC, sendo que a média do tempo de experiência era de 16 meses.

Os dados foram coletados por meio de uma escala tipo Likert, composta por 32 itens, que se destina à descrição das quatro dimensões de estilos cognitivos descritas anteriormente (Convergência-Divergência de Pensamento, Dependência-Independência de Campo, Holista-Serialista e Impulsividade-Reflexividade de Resposta). A escala foi construída e submetida a validação de definição e de constructo por Bariani (1998).

A aplicação do material foi realizada nas salas de aula dos alunos, em horários previamente agendados com seus professores.

\section{RESULTADOSEDISCUSSÃO}

Os dados foram tratados por meio de análise estatística exploratória e inferencial. Com base na validação de definição, optou-se por analisar os dados agrupados de acordo com os oito estilos cognitivos medidos. 
Tabela 1. Médias ( ), Desvio-Padrāo (DP) e resultados do Teste t de "Studentl", por estilos cognitivos, entre estudantes com e sem experiência em Programas de Iniciação Cientifica.

\begin{tabular}{|c|c|c|c|c|c|}
\hline Estilo Cognitivo & Experiência em IC & & DP & $t$ & $p$ \\
\hline \multirow{2}{*}{ Convergência } & Sem experiência & 10,2901 & 2,701 & \multirow[t]{2}{*}{3,74} & \multirow[t]{2}{*}{$0,001^{*}$} \\
\hline & Com experiència & 8,7391 & 1,888 & & \\
\hline \multirow{2}{*}{ Divergência } & Sem experiência & 14,3679 & 2,374 & \multirow[t]{2}{*}{$-3,75$} & \multirow[t]{2}{*}{$0,000^{\circ}$} \\
\hline & Com experiência & 16,2609 & 1,959 & & \\
\hline \multirow{2}{*}{ Campo Dependente } & Sem experiência & 13,9858 & 2,195 & \multirow[t]{2}{*}{2,01} & \multirow[t]{2}{*}{$0,045^{\circ}$} \\
\hline & Com experiência & 13,0435 & 2,078 & & \\
\hline \multirow{2}{*}{ Campo Independente } & Sem experiência & 12,6274 & 2,140 & \multirow[t]{2}{*}{$-0,91$} & \multirow[t]{2}{*}{0,366} \\
\hline & Com experiência & 13,0435 & 2,266 & & \\
\hline \multirow{2}{*}{ Holista } & Sem experiência & 13,5991 & 2,289 & \multirow[t]{2}{*}{$-0,20$} & \multirow[t]{2}{*}{0,843} \\
\hline & Com experiência & 13,6957 & 2,183 & & \\
\hline \multirow{2}{*}{ Serialista } & Sem experiência & 14,3231 & 2,233 & \multirow[t]{2}{*}{1,14} & \multirow[t]{2}{*}{0,255} \\
\hline & Com experiência & 13,7826 & 1,808 & & \\
\hline \multirow{2}{*}{ Impulsividade } & Sem experiência & 10,5236 & 2,678 & \multirow[t]{2}{*}{1,30} & \multirow[t]{2}{*}{0,193} \\
\hline & Com experiência & 9,7826 & 2,215 & & \\
\hline \multirow{2}{*}{ Reflexividade } & Sem experiência & 14,1014 & 2,790 & \multirow[t]{2}{*}{$-0,34$} & \multirow[t]{2}{*}{0,733} \\
\hline & Com experiência & 14,3043 & 2,512 & & \\
\hline
\end{tabular}

•Resultado significativo

Diferenças significativas $(p \leq 0,05)$ foram encontradas ao se analisarem os estilos de bolsistas e não-bolsistas de IC por meio do Teste $t$ de "Student", conforme é mostrado na Tabela 1. Estas diferenças dizem respeito aos estilos: Convergência, Divergência e Campo Dependente.

Observando-se as médias obtidas, verificase que, em relação aos estudantes sem experiência em IC, os com experiência tendem a ser significativamente menos Convergentes, mais Divergentes e menos Dependentes de Campo.

Bar-Haïm (1988) destaca que, quando os universitários têm aspirações para ocupações criativas, tal como a ciência, algumas atitudes intelectuais são altamente valorizadas, as quais, por sua vez, são influenciadas pela cognição.
De acordo com a literatura, a IC é desencadeadora de certas características e competências no sujeito que a desenvolve (Bazin, 1983; Zakon, 1989; Simão, 1994; Almeida, 1995; Bettoi, 1995e Costa, 1995). No entanto, a partir da análise dos resultados deste estudo não se pode ter certeza de que as diferenças obtidas se deram em função das atividades de IC, ou seja, não é possível afirmar que a experiência em pesquisa é a responsável pela modificação dos estilos cognitivos. Podese pensar que os estudantes buscam os programas de IC, ou são para eles escolhidos justamente devido as suas características de estilos cognitivos. Assim, neste estudo, não é dirimida a seguinte questão: Os alunos seriam "seduzidos" para a IC devido aos seus estilos cognitivos ou são as experiências nestes programas que influenciam os seus estilos? 
Tabela 2. Estilos Cognitivos e Séries: Médias ( ), Desvio-Padrão (DP), Amplitude de Variaçăo (AV) e Resultados da Análise de Variância.

\begin{tabular}{|c|c|c|c|c|c|c|}
\hline Estilo Cognitivo & Série & & DP & AV & $\mathbf{F}$ & $p$ \\
\hline \multirow{5}{*}{ Convergência } & 13 & 10,6733 & 2,8394 & $6-18$ & \multirow{5}{*}{4,4561} & \multirow{5}{*}{$0,0015^{*}$} \\
\hline & $2^{a}$ & 10,8571 & 2,5926 & $5-16$ & & \\
\hline & $3^{3}$ & 10,0667 & 2,6891 & $6-16$ & & \\
\hline & $4^{a}$ & 9,6386 & 2,4820 & $5-16$ & & \\
\hline & $5^{a}$ & 9,5467 & 2,5590 & $5-16$ & & \\
\hline \multirow{5}{*}{ Divergência } & $1^{3}$ & 14,7228 & 2,2940 & $9-19$ & \multirow{5}{*}{1,4364} & \multirow{5}{*}{0,2210} \\
\hline & $2^{i}$ & 14,2143 & 2,4547 & $7-19$ & & \\
\hline & 3 & 14,1222 & 2,4851 & $8-19$ & & \\
\hline & $4^{3}$ & 14,5060 & 2,3807 & $8-20$ & & \\
\hline & $5^{3}$ & 14,8133 & 2,2939 & $10-18$ & & \\
\hline \multirow{5}{*}{ Campo Dependente } & $1^{2}$ & 14,4158 & 2,4870 & $6-19$ & \multirow{5}{*}{3,5732} & \multirow{5}{*}{$0,0070^{\circ}$} \\
\hline & $2^{4}$ & 14,1020 & 2,0433 & $9-19$ & & \\
\hline & $3^{a}$ & 14,0000 & 1,9831 & $7-19$ & & \\
\hline & $4^{a}$ & 13,7349 & 2,1247 & $8-19$ & & \\
\hline & $5^{2}$ & 13,2267 & 2,1471 & $8-18$ & & \\
\hline \multirow{5}{*}{ Campo Independente } & $1^{32}$ & 12,9703 & 2,1469 & $8-20$ & \multirow{5}{*}{2,2700} & \multirow{5}{*}{0,0609} \\
\hline & $2^{3}$ & 12,8469 & 2,1121 & $5-18$ & & \\
\hline & $3^{a}$ & 12,5778 & 2,2234 & $7-18$ & & \\
\hline & $4^{a}$ & 12,6386 & 2,2173 & $8-17$ & & \\
\hline & $5^{a}$ & 12,0533 & 1,9305 & $7-15$ & & \\
\hline \multirow{5}{*}{ Holista } & $1^{2}$ & 13,6436 & 2,4273 & $8-18$ & \multirow{5}{*}{0,1667} & \multirow{5}{*}{0,9552} \\
\hline & $2^{a}$ & 13,6122 & 2,2550 & $8-19$ & & \\
\hline & $3^{a}$ & 13,4333 & 2,2288 & $9-19$ & & \\
\hline & $4^{a}$ & 13,6747 & 2,3484 & $8-19$ & & \\
\hline & $5^{a}$ & 13,6667 & 2,1456 & $8-19$ & & \\
\hline \multirow{5}{*}{ Serialista } & 12 & 14,5347 & 2,3476 & $8-19$ & \multirow{5}{*}{1,7271} & \multirow{5}{*}{0,1429} \\
\hline & $2^{a}$ & 14,5306 & 2,3118 & $10-20$ & & \\
\hline & $3^{a}$ & 14,4111 & 2,1924 & $8-19$ & & \\
\hline & 4 & 13,9639 & 2,1095 & $9-18$ & & \\
\hline & $5^{2}$ & 13,8933 & 1,9835 & $10-20$ & & \\
\hline \multirow{5}{*}{ Impulsividade } & 13 & 10,5347 & 2,6517 & $5-17$ & \multirow{5}{*}{0,9780} & \multirow{5}{*}{0,4192} \\
\hline & $2^{a}$ & 10,7755 & 2,7037 & $6-20$ & & \\
\hline & $3^{2}$ & 10,6111 & 2,7952 & $5-17$ & & \\
\hline & $4^{a}$ & 10,3735 & 2,7262 & $5-18$ & & \\
\hline & $5^{a}$ & 10,0133 & 2,3509 & $6-16$ & & \\
\hline \multirow{5}{*}{ Reflexividade } & $1^{3}$ & 14,5149 & 2,5908 & $4-19$ & \multirow{5}{*}{0,9534} & \multirow{5}{*}{0,4329} \\
\hline & $2^{a}$ & 13,8367 & 2,8743 & $5-20$ & & \\
\hline & $3^{a}$ & 14,1111 & 2,7209 & $8-20$ & & \\
\hline & $4^{a}$ & 13,8675 & 2,9499 & $5-20$ & & \\
\hline & $5^{a}$ & 14,2000 & 2,7460 & $8-19$ & & \\
\hline
\end{tabular}

•Resultado significativo 
Entretanto, nota-se que, de um modo geral, as características de estilos cognitivos que diferenciamos bolsistas dos não-bolsistas estão de acordo com as exigidas pelas peculiaridades das atividades de IC, destacadas por vários autores, entre elas: criatividade e curiosidade intelectual, independência cognitiva, autodisciplina e perseverança no tratamento das questões (Bachrach, 1972; Moraes, 1986; e Simão, 1994).

A Análise de Variância (8X5), considerando os oito estilos cognitivos e as séries do curso investigado, mostra dois resultados significativos $(p<0,05)$ e outro que merece ser destacado $(0,05<p<0,1)$. Estas diferenças, apresentadas na Tabela 2, indicam que da $1^{\mathrm{a}}$ à $5^{\mathrm{a}}$ série há uma propensão à diminuição dos estilos: Convergência, Campo Dependente e Campo Independente.

A diferença mais significativa, que recai no estilo Convergência, deve ser discutida. Tendo em vista a complexidade do comportamento humano e que a Psicologia não dispõe de respostas "corretas", a atuação do profissional da área exige, muitas vezes, que se lide com situações inusitadas, que nada têm de convencional. Assim, a diminuição acentuada do pensamento convergente sugere que os estudantes estão sendo preparados para atender as vicissitudes da profissão.

Pode-se supor que as vivências no curso freqüentado gerem impacto no referido estilo cognitivo, pois é função da universidade formar o profissional habilitado a lidar com múltiplas linguagens e capaz de ler as contradições da realidade (Almeida,1995).

Outro destaque é a tendência a uma significativa diminuição da Dependência de Campo e, também, decréscimo dá Independência de Campo. Há a possibilidade de ocorrer um aumento da autonomia do aluno e, portanto, diminuição da Dependência de Campo. Por outro lado, tratando-se de um curso da área de humanas, é provável que os alunos tenham uma ampla experiência em relações interpessoais e, como este tipo de experiência não é privilegiado por pessoas com Independência de Campo, este estilo tenderia à diminuição.

Deve ser destacado que podem ser encontradas pessoas que possuam tanto as características da Dependência, quanto da Independência de Campo (Witkin e Goodenough, 1991).

Além disso, como mencionado por estes mesmos autores e, também, por Thompson e Crutchlow (1993), o uso dos diferentes estilos pode depender de contextos específicos. Estudantes podem ser mais Dependentes de Campo com professores autoritários e menos com professores mais democráticos. Outros fatores podem influenciar, como regras e normas, expectativas, modos de interação e o ambiente geral de sala de aula. Os valores compartilhados por um grupo cultural também podem influenciar na modificação dos estilos.

De qualquer modo, parece que os currículos dos cursos e as experiências vividas durante os anos de universidade têm um certo impacto nos estilos cognitivos dos universitários.

\section{CONCLUSÕES}

A partir das análises realizadas, foi possível confirmar algumas suposições de estudiosos dos estilos cognitivos.

Parece que há variação na predominância de alguns estilos cognitivos dos estudantes em função das séries do curso e de terem ou não experiência em programas de IC. Pode-se, assim, dizer que não há estabilidade dos estilos cognitivos durante os anos de universidade, pois foi verificado que são passíveis de modificação, o que fortalece o modo de compreender estilos cognitivos aqui adotado como relativamente estáveis.

Não se pode, contudo, ter certeza de quais são as fontes de influência mais marcantes, se as estruturas curriculares dos 
cursos, o ambiente geral da universidade ou a convivência com outros grupos culturais. Mas presume-se que, de alguma forma, o seu status de universitário interfira em seu desenvolvimento cognitivo, pois, como salientado por Snyders (1995, p. 23): ser universitário é uma profissão de tempo integral, e que dura vários anos; penso que mesmo os seus lazeres, suas férias, pelo menos em parte, mantêm uma relação direta com seus estudos, por vias diferentes: temporadas, estágios, atividades culturais.

A possibilidade de as estruturas curriculares dos cursos terem impacto nos estilos cognitivos de seus estudantes é um forte motivo, para que sejam cuidadosamente elaboradas, tanto no que se refere aos fundamentos filosóficos e pedagógicos dos currículos, quanto no que diz respeito ao planejamento das grades curriculares e das atividades programadas no dia-a-dia das universidades.

Convém destacar que, olhar para os estilos cognitivos dos discentes, é olhar para as suas características cognitivas, ao invés de atentar apenas para suas habilidades. Recomenda-se, ainda, que esse olhar tenha uma perspectiva multidimensional, ou seja, cognitiva, afetiva, social e fisiológica.

Também, deve ser destacada a relevância das experiências vividas especificamente nos programas de IC, pois, além de serem fonte de aprendizagem acadêmica e de formação profissional, podem favorecer mudanças na estrutura cognitiva do aluno.

A IC, entendida como um objetivo educacional básico, com função formativa, parece oportunizar o desenvolvimento da flexibilidade dos estilos cognitivos, visto que, conforme sugerido por Demo (1991) e Almeida (1995), por meio da pesquisa, o aluno aprende a lidar com a provisoriedade do conhecimento, com as contradições da realidade e com múltiplas linguagens e variadas formas de percepção.

Um grande número de autores discute enfaticamente sobre os benefícios do ensino superior para o estudante, ou sobre a importância do desenvolvimento no aluno de uma atitude científica, que o leve a aprender a aprender, a produzir conhecimento próprio, mas pouco é abordado no Brasil sobre o impacto das experiências curriculares e das vividas nos programas de IC sobre a estrutura cognitiva dos universitários.

Espera-se que este trabalho sirva para encorajar outros pesquisadores a dar continuidade a esta linha de estudo e para que professores e estudantes tirem proveito dos conhecimentos aqui apresentados, no sentido da otimização das relações interpessoais estabelecidas entre eles, das suas estruturas cognitivas e do processo ensino-aprendizagem como um todo.

\section{REFERÊNCIAS}

ABOUSERIE, R; Moss, D.;\& Barasi, S. (1992) Cognitive Style, Gender, Altitude toward Computer-Assisted Learning and Academic Achievement. Educational Studies, 18 (2), 151-160.

ALLINSON, C. W.; Hayes, J.; \& Davis, A. (1994) Matching the Cognitive Style of Management Students and Teachers: A Preliminary Study. Perceptual and Motor Skills, 79 (3, pt 1), 1256-1258.

ALMEIDA, L. M. do A. C. (1995). Sobre a Iniciação Científica, ou sobre a Difícil Tarefa de Formar Profissionais Críticos e Autônomos. Anais do I Encontro de Iniciação Científica da USF, 22-24, Universidade São Francisco.

AZZI, R. G.(1993). Pesquisa em Educação e Psicologia: Identificação de Condições que Favorecem sua Ocorrência na Universidade. Tese de Doutorado, FE, UNICAMP.

BACHRACH, A. J. (1972). Introdução à Pesquisa Psicológica. SP, Helder. (Tradução de Geraldina Porto Witter, do original em inglês.) 
BAR-HAiM, G. (1988). Problem-solvers and problem-identifiers: The making of research styles. International Journal of Science Education, 10 (2),135-150.

BAR-HAïM, G. (1984). Styles of research and research self-images of academics in four socio-behavioral fields. Social Science Information -sur les sciences sociales, 23 (6),1007- 028.

BARIANI, I. C. D. (1998) Estilos Cognitivos de Universitários e Iniciação Científica. Tese de Doutorado, FE-UNICAMP.

BARIANI, I. C. D. e Dimárzio, M. K. ( 1997) Perspectivas da Iniciação Científica por Estudantes Bolsistas de I. C. XXVII Reunião Anual de Psicologia, SBP, Ribeirão Preto, SP (Não Publicado).

BAZIN, M. J.(1983) O que é Iniciação Científica.Revista de Ensino de Física, 5 (1), 81- 88.

BETTOI, S. M. (1995) O Pesquisar na Graduação: A Palavra do Aluno de Psicologia sobre as Condições Presentes na sua Vida Acadêmica. Dissertação de Mestrado, PUC-SP.

COSTA, D. (1995). A Importância do Programa de Iniciação Científica para a Formação de Pesquisadores. Anais do I Encontro de Iniciação Científica da USF, 19-20, Universidade São Francisco.

DEMO, P. (1991). Qualidade e Modernidade da Educação Superior: Discutindo questões de qualidade, eficiência e pertinência. Educação Brasileira, 13 (27), 35-80.

GIL, A. C. (1987) Como Elaborar Projetos de Pesquisa. SP, Atlas.

HALPIN, G.; \& Peterson, H. (1986) Accomodating Instruction to Learners 'Field Independence/ Dependence:A Study of Effects on Achievement and Attitudes. Perceptual and Motor Skills, 62 (2), 967-974.

KERLINGER, F. N. (1980) Metodologia da Pesquisa em Ciências Sociais: Um tratamento conceitual. SP, EPU-EDUSP (Tradução de Helena Mendes Rotundo, do original em inglês.)

MARQUES, E. A. e Gols (1989) Ensino e Pesquisa na Universidade: Questão de Lei ou de Visão de Mundo? Caderno de Pesquisa, 69, 5-16.

MESSICK, S. (1984) The Nature of Cognitive Styles: Problems and Promise in Educational Practice. Educational Psychology, 19 (2),59-74.

MORAES, I. N. (1986). Perfil da Universidade. SP, Pioneira.

PALMER, J. (1991). Scientists and Information: II. Personal Factors In Information Behaviour. Journal of Documentation, 47 (3), 254-275.

RIDING, R. \& Cheema, I. (1991). Cognitive Styles - an over view and integration. Educational Psychology, 11 (3/4), 193-215.

RIDING, R. \& Sadler-Smith, E. (1992). Type of Instructional Material, Cognitive Style and Learning Performance. Educational Studies, 18 (3), 323-340.

RIDING, R. \& Wright, M. (1995). Cognitive Style, Personal Characteristics and Harmony in Student Flats. Educational Psychology, 15 (3), 337-349.

SIMÃO, L. M. (1994). Considerações sobre formas de relação entre Iniciação Científica e Pós-Graduação. V Simpósio de Pesquisa e Intercâmbio Científico, ANPPEP (Mimeo).

SNYDERS, G. (1995). Feliz na Universidade: estudo a partir de algumas biografias. Rio de Janeiro, Paz e Terra. (Tradução de Antonio de Padua Danesi, do original em francês.)

THOMPSON, C. \& Crutchlow, E. (1993). Learning Style Research: A Critical Review of Ihe Literature and Implications for 
Nursing Education. Journal of Professional Nursing, 9 (1), 34-40.

WITKIN,H. A. \& Goodenough, D. R. (1991). Estilos Cognitivos: Natureza y Orígines. Madrid, Ediciones Pirámide. (Tradução de Pilar Sánchez Lópes e Ángeles Quiroga, do original em inglês para o espanhol.)

WOOD, F. (1990) Factores Influencing Research Performance of University
61 Academic Staff. Higher Education, 19 (1),81-100.

WOOTEN, K. C. \& Barner, B. O. (1994) The Influence of Cognitive Style upon Work Environment Preferences. Perceptual and Motor Skills, 79, 307-314.

ZAKON, A. (1989). Qualidades Desejáveis na Inclinação Científica. Ciência e Cultura, 41 (9), 868-877. 